\title{
Generalized Buneman Dispersion Relation in the Longitudinally Dominated Magnetic Field
}

\author{
Martin Bohata, ${ }^{1}$ David Břeň, ${ }^{2}$ and Petr Kulhánek ${ }^{1}$ \\ ${ }^{1}$ Faculty of Electrical Engineering, Czech Technical University in Prague, Technická 2, 16627 Prague 6, Czech Republic \\ ${ }^{2}$ Faculty of Nuclear Sciences and Physical Engineering, Czech Technical University in Prague, Břehová 7 , \\ 11519 Prague 1, Czech Republic
}

Correspondence should be addressed to Petr Kulhánek, kulhanek@fel.cvut.cz

Received 5 September 2011; Accepted 13 October 2011

Academic Editor: S. Chromik

Copyright (c) 2011 Martin Bohata et al. This is an open access article distributed under the Creative Commons Attribution License, which permits unrestricted use, distribution, and reproduction in any medium, provided the original work is properly cited.

\begin{abstract}
The generalized Buneman dispersion relation for two-component plasma is derived in the case of nonzero pressure of both plasma components and longitudinally dominated magnetic field. The derived relation is also valid for other field configurations mentioned in the paper. It can be useful in a variety of plasma systems, for example, in the analyses of plasma jet penetrating into background plasma, in beam-plasma physics, and in tests of various magnetohydrodynamics (MHD) and hybrid numerical codes designed for the magnetized plasmas.
\end{abstract}

\section{Introduction}

The two stream instabilities are the most common instabilities in plasmas. Usually plasma consists of two or more kinds of particles moving with different velocities. Under suitable conditions the two-stream instability can occur and particle energy is transferred into the energy of plasma wave excitation [1-3]. The plasma subcomponents with nonzero initial velocity are thermalized and the plasma temperature increases.

The two-stream instability is observed in plasma jets injected into the background plasma, in beam-plasma and beam-target configurations (e.g., in fusion experiments), and in some other configurations in which the different species have different drift velocities.

For cold unmagnetized plasma with two counter streaming components the problem has been solved by Buneman in his pioneering works in late fifties $[4,5]$. He found the fastest stable drift velocity for the electrons and conditions under which the energy is dissipated into the instabilities. The Buneman instability was studied in a variety of experiments, for example, [6].

The amplitude saturation of Buneman instability was discussed in detail much later by Hirose in [7]. The role of the Buneman instability in reconnection processes is very interesting, especially in the vicinity of the plasma neutral sheet [8]. In nonlinear mode the Buneman instability can be the starting mechanism for the onset of ion-acoustic instability [9]. The Buneman instability has many modifications (e.g., two symmetric opposite beams, beam-target instability, etc.) and several related classes of instabilities. First of them is the Farley-Buneman Instability, which develops if electron and ion velocities differ by more than ion acoustic speed. This instability is wellknown in the ionosphere region $\mathrm{E}$ [10]. Above some critical value of the current the Buneman instability changes into the Pierce instability which causes sudden nonoscillatory potential drop leading to the double layer formation and capture of ions in the potential well [11]. In the case of anisotropic velocity distribution the socalled Weibel instability develops, which was described in the same time period as the Buneman instability [12]. All these types of instabilities dissipate the particle energy into the plasma waves or other plasma disturbances. In some sense all these instabilities are opposite to the well-known Landau damping predicted in 1946, in which particles can gain the energy from the wave modes [13]. Details of the variety of Buneman-like instabilities can be found in $[1,14,15]$.

Buneman-like instabilities are very frequently used as test examples for various MHD and hybrid numerical electrostatic codes $[16,17]$. This is advantageous, as the 
plasma behavior in corresponding regimes is wellknown both theoretically and experimentally. The generalized dispersion relation derived later in this paper could be helpful for similar tests in magnetized plasmas.

\section{Basic Assumptions}

Let us assume a two-component unbounded plasma under the following conditions.

(1) Plasma is described by nonrelativistic set of equations:

$$
\begin{gathered}
\frac{\partial n_{\alpha}}{\partial t}+\nabla \cdot\left(n_{\alpha} \mathbf{u}_{\alpha}\right)=0 \\
m_{\alpha} n_{\alpha} \frac{\partial \mathbf{u}_{\alpha}}{\partial t}+m_{\alpha} n_{\alpha}\left(\mathbf{u}_{\alpha} \cdot \nabla\right) \mathbf{u}_{\alpha}=-\nabla p_{\alpha}+q_{\alpha} n_{\alpha}\left(\mathbf{E}+\mathbf{u}_{\alpha} \times \mathbf{B}\right) \\
\nabla \times \mathbf{E}=-\frac{\partial \mathbf{B}}{\partial t}, \\
\frac{1}{\varepsilon_{0} \mu_{0}} \nabla \times \mathbf{B}=\frac{1}{\varepsilon_{0}} \mathbf{j}+\frac{\partial \mathbf{E}}{\partial t} ; \quad \mathbf{j}=\sum_{\alpha=1}^{2} q_{\alpha} n_{\alpha} \mathbf{u}_{a} \\
p_{\alpha}=K_{\alpha} n_{\alpha}^{\gamma_{\alpha}}
\end{gathered}
$$

Expression (1) represents continuity equations, (2) equations of motion, (3) and (4) Maxwell equations for the electric and magnetic fields, and (5) polytrophic formulas for the pressures. Index $\alpha$ denotes the two plasma components (e.g., electrons and ions).

(2) Perturbations of all the variables are considered to be linear, that is,

$\psi_{\alpha}(t, \mathbf{x})=\psi_{\alpha}^{(0)}+\lambda \psi_{\alpha}^{(1)}(\omega, \mathbf{k}) \mathrm{e}^{i[\mathbf{k} \cdot \mathbf{x}-\omega t]}+O\left(\lambda^{2}\right)$.

(3) All the zero-order solutions $\psi_{\alpha}^{(0)}$ are constant in time and space. To ensure this assumption we will suppose that magnetic field component perpendicular to the direction of particle stream motion is weak (magnetic field is longitudinally dominated for both plasma species); that is, the Larmor radii are much greater than arbitrary watched plasma size $L$, and therefore gyration is negligible:

$$
\frac{m_{\alpha} u_{\alpha \perp}^{(0)}}{q_{\alpha} B_{\perp}^{(0)}} \gg L
$$

The other possibility is to meet the set of conditions resulting from (1)-(5) and Maxwell equation $\operatorname{div} \mathbf{D}=\rho$ (where $\rho$ is the charge density):

$$
\begin{gathered}
\mathbf{E}^{(0)}+\mathbf{u}_{\alpha}^{(0)} \times \mathbf{B}^{(0)}=\mathbf{0}, \\
\rho^{(0)}=\sum_{\alpha=1}^{2} q_{\alpha}^{(0)} n_{\alpha}^{(0)}=0, \\
\mathbf{j}^{(0)}=\sum_{\alpha=1}^{2} q_{\alpha}^{(0)} n_{\alpha}^{(0)} \mathbf{u}_{\alpha}^{(0)}=\mathbf{0} .
\end{gathered}
$$

For $\mathbf{E}^{(0)}=0$ is the first condition in (8) akin to (7).

\section{Linearization of the Equations for Arbitrary Frequencies}

Perturbing the whole set of (1)-(5) according to (6), we obtain in the first order of the parameter $\lambda$ a linearized set of algebraic equations:

$$
\begin{gathered}
\Omega_{\alpha} n_{\alpha}^{(1)}-n_{\alpha}^{(0)} \mathbf{k} \cdot \mathbf{u}_{\alpha}^{(1)}=0 \\
-i m_{\alpha} n_{\alpha}^{(0)} \Omega_{\alpha} \mathbf{u}_{\alpha}^{(1)}=-i \mathbf{k} p_{\alpha}^{(1)}+q_{\alpha} n_{\alpha}^{(0)} \mathbf{E}^{(1)}+q_{\alpha} n_{\alpha}^{(1)} \mathbf{E}^{(0)} \\
\quad+q_{\alpha} n_{\alpha}^{(0)} \mathbf{u}_{\alpha}^{(0)} \times \mathbf{B}^{(1)}+q_{\alpha} n_{\alpha}^{(0)} \mathbf{u}_{\alpha}^{(1)} \times \mathbf{B}^{(0)} \\
+q_{\alpha} n_{\alpha}^{(1)} \mathbf{u}_{\alpha}^{(0)} \times \mathbf{B}^{(0)} \\
\quad \mathbf{B}^{(1)}=\frac{1}{\omega} \mathbf{k} \times \mathbf{E}^{(1)} \\
-i \omega \mathbf{E}^{(1)}=i c^{2} \mathbf{k} \times \mathbf{B}^{(1)}-\frac{\mathbf{j}^{(1)}}{\varepsilon_{0}} \\
\mathbf{j}^{(1)}=\sum_{\alpha=1}^{2}\left(q_{\alpha} n_{\alpha}^{(0)} \mathbf{u}_{\alpha}^{(1)}+q_{\alpha} n_{\alpha}^{(1)} \mathbf{u}_{\alpha}^{(0)}\right) \\
p_{\alpha}^{(1)}=m_{\alpha} c_{s \alpha}^{2} n_{\alpha}^{(1)}
\end{gathered}
$$

where we have denoted the Doppler shifted frequency:

$$
\Omega_{\alpha}=\omega-\mathbf{k} \cdot \mathbf{u}_{\alpha}^{(0)},
$$

sound velocities:

$$
c_{s \alpha}=\sqrt{\frac{\gamma_{\alpha} k_{B} T_{\alpha}}{m_{\alpha}}},
$$

and the velocity of light:

$$
c=\sqrt{\frac{1}{\varepsilon_{0} \mu_{0}}} .
$$

Substituting (11) into (12) we achieve the equation for the electric field perturbation in the following form:

$$
\mathbb{M} \mathbf{E}^{(1)}=-i \omega \mu_{0} \mathbf{j}^{(1)} ; \quad \mathbb{M}=\left(\frac{\omega^{2}}{c^{2}}-k^{2}\right) \square+\mathbf{k} \otimes \mathbf{k},
$$

where $\square$ denotes the identity matrix. The matrix $\mathbb{M}$ can be easily inverted as

$$
\mathbb{M}^{-1}=\frac{\square-\left(c^{2} / \omega^{2}\right) \mathbf{k} \otimes \mathbf{k}}{\omega^{2} / c^{2}-k^{2}},
$$

and the electric field perturbation becomes

$$
\mathbf{E}^{(1)}=-\frac{i \omega \mu_{0}}{\omega^{2} / c^{2}-k^{2}}\left[\mathbf{j}^{(1)}-\frac{c^{2}}{\omega^{2}}\left(\mathbf{k} \cdot \mathbf{j}^{(1)}\right) \mathbf{k}\right] .
$$

Now the pressure perturbations $p_{\alpha}^{(1)}$ can be derived from (14), perturbations of concentrations $n_{\alpha}^{(1)}$ from the continuity equations of (9), magnetic field perturbation $\mathbf{B}^{(1)}$ 
from (11), electric field perturbation $\mathbf{E}^{(1)}$ from (20), and electric current perturbation $\mathbf{j}^{(1)}$ from (13). After substitution of all these perturbations into the equations of motion (10) we finally get

$$
\begin{aligned}
& \Omega_{\alpha}^{2} \mathbf{u}_{\alpha}^{(1)}= \frac{i}{m_{\alpha}}\left(\mathbf{k} \cdot \mathbf{u}_{\alpha}^{(1)}\right) \mathbf{F}_{\alpha}^{(0)}+c_{s \alpha}^{2}\left(\mathbf{k} \cdot \mathbf{u}_{\alpha}^{(1)}\right) \mathbf{k} \\
&+i \xi_{\alpha} \Omega_{\alpha} \mathbf{u}_{\alpha}^{(1)} \times \mathbf{B}^{(0)}+\xi_{\alpha} \Omega_{\alpha} \frac{\mu_{0} c^{2}}{\omega^{2}-c^{2} k^{2}} \\
& \times\left[\Omega_{\alpha} \mathbf{j}^{(1)}+\left(\mathbf{u}_{\alpha}^{(0)} \cdot \mathbf{j}^{(1)}\right) \mathbf{k}-\frac{c^{2}}{\omega}\left(\mathbf{k} \cdot \mathbf{j}^{(1)}\right) \mathbf{k}\right], \\
& \alpha=1,2,
\end{aligned}
$$

where

$$
\mathbf{j}^{(1)}=\sum_{\alpha=1}^{2}\left[q_{\alpha} n_{\alpha}^{(0)}\left(\mathbf{u}_{\alpha}^{(1)}+\frac{\mathbf{k} \cdot \mathbf{u}_{\alpha}^{(1)}}{\omega-\mathbf{k} \cdot \mathbf{u}_{\alpha}^{(0)}} \mathbf{u}_{\alpha}^{(0)}\right)\right] .
$$

In expression (21) we have denoted

$$
\xi_{\alpha}=\frac{q_{\alpha}}{m_{\alpha}}, \quad \mathbf{F}_{\alpha}^{(0)}=q_{\alpha}\left[\mathbf{E}^{(0)}+\mathbf{u}_{\alpha}^{(0)} \times \mathbf{B}^{(0)}\right] .
$$

The quantities $\xi_{\alpha}, \mathbf{F}_{\alpha}^{(0)}$ symbolize specific charge and zeroorder Lorentz force, respectively. In case of two-component plasma the expression (21) represents 6 equations for velocity perturbations. Both (21) and (22) can be simply written for more than two plasma components, in which case $\alpha=$ $1, \ldots, n$.

\section{Generalized Dispersion Relation in Low-Frequency Limit}

In low-frequency limit $\omega \ll c k$, (21) and (22) give us

$$
\begin{aligned}
\Omega_{\alpha}^{2} \mathbf{u}_{\alpha}^{(1)}= & \frac{i}{m_{\alpha}}\left(\mathbf{k} \cdot \mathbf{u}_{\alpha}^{(1)}\right) \mathbf{F}_{\alpha}^{(0)} \\
& +\left(c_{s \alpha}^{2}+\frac{\omega_{p \alpha}^{2}}{k^{2}}\right)\left(\mathbf{k} \cdot \mathbf{u}_{\alpha}^{(1)}\right) \mathbf{k} \\
& +\frac{\xi_{\alpha} \Omega_{\alpha}}{\xi_{\beta} \Omega_{\beta}} \frac{\omega_{p \beta}^{2}}{k^{2}}\left(\mathbf{k} \cdot \mathbf{u}_{\beta}^{(1)}\right) \mathbf{k} \\
& +i \xi_{\alpha} \Omega_{\alpha} \mathbf{u}_{\alpha}^{(1)} \times \mathbf{B}^{(0)},
\end{aligned}
$$

where the indices $\alpha$ and $\beta$ are different, that is, $\beta \neq \alpha$, and furthermore we have designated square of the plasma frequency as

$$
\omega_{p \alpha}^{2}=\frac{n_{\alpha}^{(0)} q_{\alpha}^{2}}{m_{\alpha} \varepsilon_{0}} .
$$

The linearized set of (24) can be rewritten in the following form:

$$
\begin{aligned}
& \mathbb{A} \mathbf{u}_{1}^{(1)}+\mathbb{B} \mathbf{u}_{2}^{(1)}=0, \\
& \mathbb{C} \mathbf{u}_{1}^{(1)}+\mathbb{D} \mathbf{u}_{2}^{(1)}=0,
\end{aligned}
$$

or simply

$$
\left(\begin{array}{ll}
\mathbb{A} & \mathbb{B} \\
\mathbb{C} & \mathbb{D}
\end{array}\right)\left(\begin{array}{l}
\mathbf{u}_{1}^{(1)} \\
\mathbf{u}_{2}^{(1)}
\end{array}\right)=0,
$$

where

$$
\begin{gathered}
\mathbb{A}=-\Omega_{1}^{2} \square+\frac{i}{m_{1}} \mathbf{F}_{1}^{(0)} \otimes \mathbf{k} \\
+\left(c_{s 1}^{2}+\frac{\omega_{p 1}^{2}}{k^{2}}\right) \mathbf{k} \otimes \mathbf{k}+i \xi_{1} \Omega_{1} \boldsymbol{\varepsilon} \cdot \mathbf{B}^{(0)} \\
\mathbb{B}=\frac{\xi_{1} \Omega_{1} \omega_{p 2}^{2}}{\xi_{2} \Omega_{2} k^{2}} \mathbf{k} \otimes \mathbf{k} \\
\mathbb{C}=\frac{\xi_{2} \Omega_{2} \omega_{p 1}^{2}}{\xi_{1} \Omega_{1} k^{2}} \mathbf{k} \otimes \mathbf{k}
\end{gathered}
$$

$$
\begin{aligned}
\mathbb{D}= & -\Omega_{2}^{2} \rrbracket+\frac{i}{m_{2}} \mathbf{F}_{2}^{(0)} \otimes \mathbf{k} \\
& +\left(c_{s 2}^{2}+\frac{\omega_{p 2}^{2}}{k^{2}}\right) \mathbf{k} \otimes \mathbf{k}+i \xi_{2} \Omega_{2} \boldsymbol{\varepsilon} \cdot \mathbf{B}^{(0)} .
\end{aligned}
$$

The symbol $\boldsymbol{\varepsilon}$ stands for the Levi-Civita tensor. The set of equations of (27) will have nontrivial solution if the determinant of the matrix will be zero:

$$
\operatorname{det}\left(\begin{array}{ll}
\mathbb{A} & \mathbb{B} \\
\mathbb{C} & \mathbb{D}
\end{array}\right)=0 .
$$

From definitions (29) and (30) it is obvious that

$$
\begin{aligned}
& \operatorname{det} \mathbb{B}=0, \\
& \operatorname{det} \mathbb{C}=0 .
\end{aligned}
$$

Relation (32) can be simply solved if either $\operatorname{det} \mathbb{A} \neq 0$ or $\operatorname{det} \mathbb{D} \neq 0$. In the first case we can calculate from the set of equations of $(26)$

$$
\mathbf{u}_{1}^{(1)}=-\mathbb{A}^{-1} \mathbb{B} \mathbf{u}_{2}^{(1)}
$$

and substitute this expression into the remaining equation in (26):

$$
\left(\mathbb{D}-\mathbb{C A}^{-1} \mathbb{B}\right) \mathbf{u}_{2}^{(1)}=0 .
$$

Nontrivial solution will exist if

$$
\operatorname{det}\left(\mathbb{D}-\mathbb{C A}^{-1} \mathbb{B}\right)=0 .
$$

The second possibility det $\mathbb{D} \neq 0$ leads us to a similar condition:

$$
\operatorname{det}\left(\mathbb{A}-\mathbb{B D}^{-1} \mathbb{C}\right)=0
$$


After straightforward but rather toilsome calculation both (36) and (37) provide the same dispersion relation:

$$
\begin{gathered}
\operatorname{det} \mathbb{A} \operatorname{det} \mathbb{D}-\frac{\omega_{p 1}^{2} \omega_{p 2}^{2}}{k^{4}}\left[\Omega_{1}^{4} k^{2}-\Omega_{1}^{2} \omega_{c 1}^{2}\left(\mathbf{e}_{B} \cdot \mathbf{k}\right)^{2}\right] \\
\times\left[\Omega_{2}^{4} k^{2}-\Omega_{2}^{2} \omega_{c 2}^{2}\left(\mathbf{e}_{B} \cdot \mathbf{k}\right)^{2}\right]=0,
\end{gathered}
$$

where

$$
\omega_{c \alpha}=\frac{q_{\alpha} B^{(0)}}{m_{\alpha}}
$$

are the cyclotron frequencies of both species. The unit vector in the zero-order magnetic field direction is designated as

$$
\mathbf{e}_{B}=\frac{\mathbf{B}^{(0)}}{B^{(0)}} .
$$

Evaluation of $\operatorname{det} \mathbb{A}$ gives us

$$
\begin{aligned}
\operatorname{det} \mathbb{A}= & \Omega_{1}^{6}-\Omega_{1}^{4}\left[i\left(\frac{\mathbf{F}_{1}^{(0)}}{m_{1}} \cdot \mathbf{k}\right)+\left(c_{s 1}^{2} k^{2}+\omega_{p 1}^{2}\right)+\omega_{c 1}^{2}\right] \\
& -\Omega_{1}^{3} \omega_{c 1}\left(\frac{\mathbf{F}_{1}^{(0)}}{m_{1}} \times \mathbf{k}\right) \cdot \mathbf{e}_{B} \\
& +\Omega_{1}^{2} \omega_{c 1}^{2}\left(\mathbf{k} \cdot \mathbf{e}_{B}\right) \\
& \times\left[i \frac{\mathbf{F}_{1}^{(0)} \cdot \mathbf{e}_{B}}{m_{1}}+\left(c_{s 1}^{2} k^{2}+\omega_{p 1}^{2}\right) \frac{\mathbf{k} \cdot \mathbf{e}_{B}}{k^{2}}\right] .
\end{aligned}
$$

Interchanging the indices 1 and 2 in $\operatorname{det} \mathbb{A}$ leads us to the value of the det $\mathbb{D}$. Hence the nontrivial solution of (27) exists if

$$
\begin{aligned}
\prod_{\alpha=1}^{2}\left\{\Omega_{\alpha}^{4}\right. & -\Omega_{\alpha}^{2}\left[i \frac{\mathbf{F}_{\alpha}^{(0)} \cdot \mathbf{k}}{m_{\alpha}}+\left(c_{s \alpha}^{2} k^{2}+\omega_{p \alpha}^{2}\right)+\omega_{c \alpha}^{2}\right] \\
& -\frac{\Omega_{\alpha} \omega_{c \alpha}}{m_{\alpha}}\left(\mathbf{F}_{\alpha}^{(0)} \times \mathbf{k}\right) \cdot \mathbf{e}_{B} \\
& \left.+\omega_{c \alpha}^{2}\left(\mathbf{k} \cdot \mathbf{e}_{B}\right)\left[i \frac{\mathbf{F}_{\alpha}^{(0)} \cdot \mathbf{e}_{B}}{m_{\alpha}}+\left(c_{s \alpha}^{2} k^{2}+\omega_{p \alpha}^{2}\right) \frac{\mathbf{k} \cdot \mathbf{e}_{B}}{k^{2}}\right]\right\} \\
& -\prod_{\alpha=1}^{2} \frac{\omega_{p \alpha}^{2}}{k^{2}}\left[\Omega_{\alpha}^{2} k^{2}-\omega_{c \alpha}^{2}\left(\mathbf{e}_{B} \cdot \mathbf{k}\right)^{2}\right]=0 .
\end{aligned}
$$

In the case of $\mathbf{E}^{(0)}=0, \mathbf{B}^{(0)}=0$, and cold plasma limit $c_{s \alpha}=0$, this relation becomes the well-known Buneman dispersion relation:

$$
\sum_{\alpha=1}^{2} \frac{\omega_{p \alpha}^{2}}{\left(\omega-\mathbf{k} \cdot \mathbf{u}_{\alpha}^{(0)}\right)^{2}}=1
$$

\section{Some Remarks to the Policy of Computation of the Dispersion Relation for Arbitrary Frequencies}

Without the low-frequency limit request, (21) can be rewritten in the matrix form (27) again:

$$
\left(\begin{array}{ll}
\mathbb{A} & \mathbb{B} \\
\mathbb{C} & \mathbb{D}
\end{array}\right)\left(\begin{array}{l}
\mathbf{u}_{1}^{(1)} \\
\mathbf{u}_{2}^{(1)}
\end{array}\right)=0 .
$$

The matrices $\mathbb{A}, \mathbb{B}, \mathbb{C}$, and $\mathbb{D}$ differ from the low-frequency matrixes (28)-(31) and can be determined from (21). The matrix $B$ has determinant

$$
\operatorname{det} \mathbb{B}=\left(\frac{\xi_{1}}{\xi_{2}}\right)^{3} \frac{\omega_{p 2}^{6}}{\left(\omega^{2}-c^{2} k^{2}\right)^{2}} \frac{\Omega_{1}^{5}}{\Omega_{2}},
$$

which is now nonzero and finite with exception of the discrete frequencies $\mathbf{k} \cdot \mathbf{u}_{1}^{(0)}, \mathbf{k} \cdot \mathbf{u}_{2}^{(0)}$, and $c k$. Excusing these frequencies the inverse matrix of $\mathbb{B}$ can be calculated as

$$
\begin{aligned}
\mathbb{B}^{-1}=A[ & \left(\Omega_{1} \Omega_{2}+\Omega_{1} \mathbf{u}_{2}^{(0)} \cdot \mathbf{k}+\Omega_{2} \mathbf{u}_{1}^{(0)} \cdot \mathbf{k}\right. \\
& \left.+\left(\mathbf{u}_{1}^{(0)} \cdot \mathbf{u}_{2}^{(0)}-c^{2}\right) k^{2}\right) \square-\Omega_{1} \mathbf{u}_{2}^{(0)} \otimes \mathbf{k}-\Omega_{2} \mathbf{k} \otimes \mathbf{u}_{1}^{(0)} \\
& \left.-\left(\mathbf{u}_{1}^{(0)} \cdot \mathbf{u}_{2}^{(0)}-c^{2}\right) \mathbf{k} \otimes \mathbf{k}+\left(\mathbf{u}_{1}^{(0)} \times \mathbf{k}\right) \otimes\left(\mathbf{k} \times \mathbf{u}_{2}^{(0)}\right)\right],
\end{aligned}
$$

where

$$
A=\frac{1}{\operatorname{det} \mathbb{B}}\left(\frac{\xi_{1}}{\xi_{2}} \frac{\omega_{p 2}^{2}}{\omega^{2}-c^{2} k^{2}}\right)^{2} \frac{\Omega_{1}^{3}}{\Omega_{2}} .
$$

From matrix equation (44) it follows

$$
\begin{gathered}
\mathbf{u}_{2}^{(1)}=\mathbb{B}^{-1} \mathbb{A} \mathbf{u}_{1}^{(1)}, \\
\left(\mathbb{C}+\mathbb{D} \mathbb{B}^{-1} \mathbb{A}\right) \mathbf{u}_{1}^{(1)}=0 .
\end{gathered}
$$

Dispersion relation for arbitrary frequencies (with exception of the frequencies mentioned above) is represented by the following condition:

$$
\operatorname{det}\left(\mathbb{C}+\mathbb{D} \mathbb{B}^{-1} \mathbb{A}\right)=0 .
$$

Analytical calculation is difficult due to the enormous number of terms in this relation, and numerical approach seems to be more advantageous in this case. Maybe some numerical methods could be also applied directly to the set of equations of (21).

\section{Results}

The new dispersion relation (42) was carefully analyzed numerically. The complex roots were found by the HubbardSchleicher-Sutherland algorithm [18]. The code was written in Compaq Visual FORTRAN 6.6.C3. Here we will discuss some results for two identical opposite beams, because they can be easily compared to the well-known 


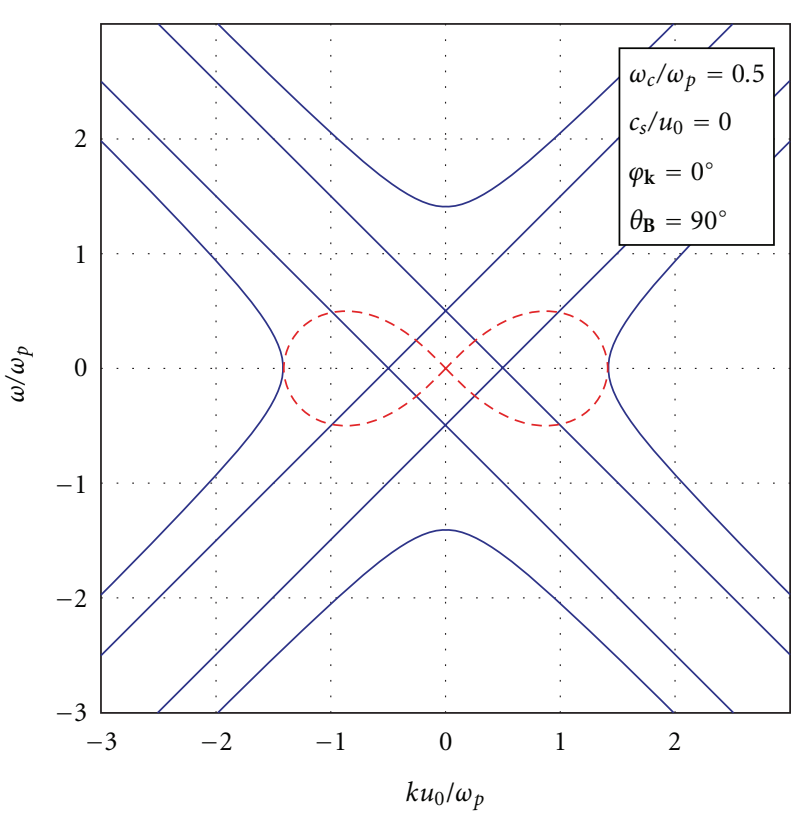

(a)

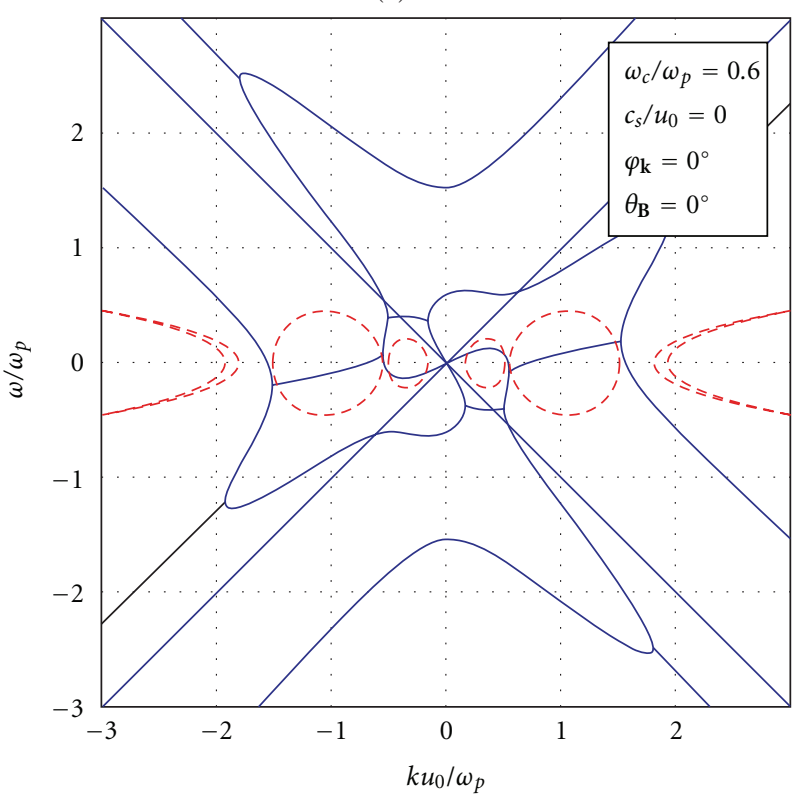

(c)

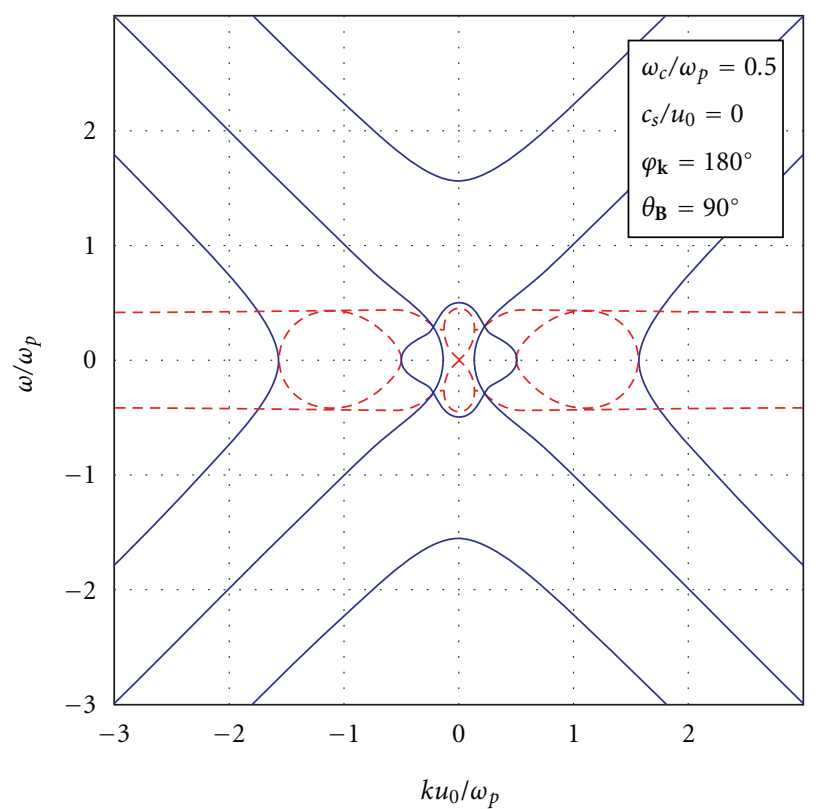

(b)

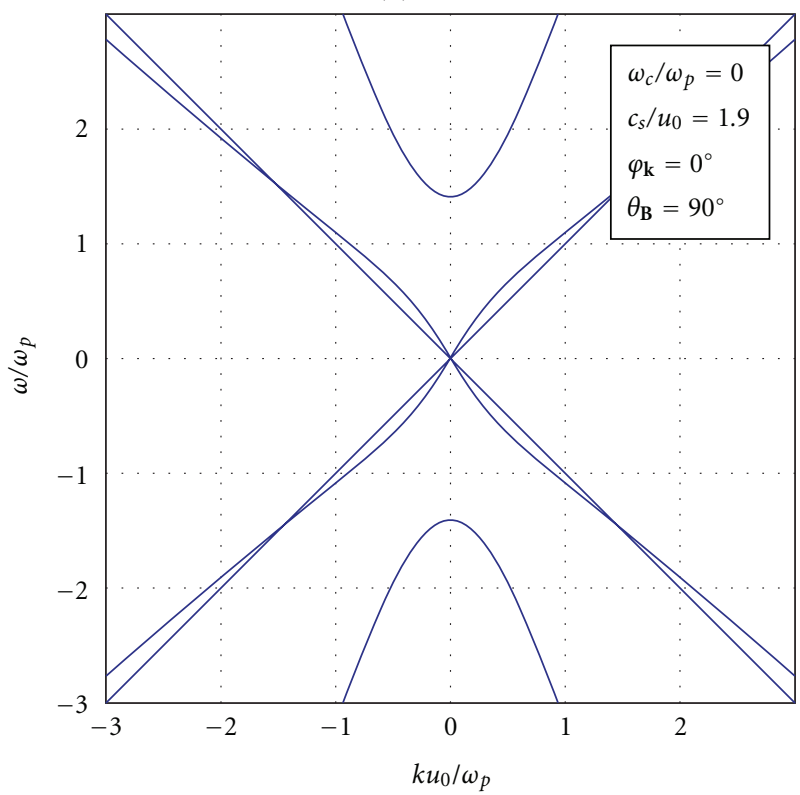

(d)

FIgURE 1: Numerical solution of the dispersion relation (42) for various parameters. Two opposite beams of the same particles were treated. The dashed red lines represent imaginary parts of the relation, and the solid blue lines the real parts.

Buneman solution. All the variables were converted into the nondimensional form. Both beams were oriented in the direction of the $x$-axis, magnetic field in the $(x, z)$ plane; that is, $B_{z}$ describes the perpendicular component of the field and $B_{x}$ the longitudinal one. There were 5 main input parameters: non-dimensional magnetic field induction as the ratio $\omega_{c} / \omega_{p}$, nondimensional sound velocity in the combination $c_{s} / u_{0}$ ( $u_{0}$ being the beam velocity), and three directional characteristics - the angle between the magnetic field and the $z$-axis $\theta_{\mathrm{B}}$, the angle between the wave vector and the $z$-axis is denoted as $\theta_{\mathbf{k}}$, and the $(x, y)$ azimuth of the wave vector $\varphi_{\mathbf{k}}$. The dispersion relation provides many new features, especially new imaginary branches of the frequency, which imply new types of instabilities. The principal results can be divided into several points.

(1) Minimal changes (compared to the Buneman solution) occur in the presence of the longitudinal magnetic field applied in the direction of the wave vector describing the perturbation. All the dispersion relation branches are depicted in the graph on Figure 1(a). The typical "8"-shaped imaginary branch (well known from the Buneman solution) remains unchanged in the $(\omega, k)$ plane as well as the 
hyperbola-like real branches. But there are four new linear dependencies $\omega= \pm \omega_{c} \pm u_{0} k$. These lines correspond to the Doppler-shifted cyclotron motion of the charged particles.

(2) If the magnetic field vector is opposite to the wave vector describing the perturbation, the picture changes completely; see Figure 1(b). Two of the cyclotron lines disappear and the two residual lines change into hyperbola-like shape. Furthermore the portrait of the imaginary parts of the dispersion relation consists of 7 branches corresponding to the new types of instabilities. The original " 8 "-like imaginary branch broke into two parts and new " 8 "-like imaginary curve appears in the vertical direction of the $(\omega, k)$ plane. Furthermore the dispersion relation gives 4 new imaginary branches, which are only very weakly dependent on the perturbation wavelength.

(3) The most complicated picture can be seen in the case of perpendicular magnetic field; see Figure 1(c). The original hyperbola-like real parts bifurcate two times in a sequence. Near the origin of the $(\omega, k)$ plane there are 4 closed imaginary part branches and the bifurcating real part branches. The only "Doppler" curves $\omega= \pm u_{0} k$ remained unchanged. The behavior of the plasma in this region of parameters seems to be very interesting and deserves detailed experimental investigation. Let us remark that stronger perpendicular fields are not compatible with the assumptions of our analysis.

(4) The pressure term also influences the dispersion relation solutions. The imaginary parts are present only in the supersonic velocity of the beams. If the velocity is subsonic, the imaginary solutions completely disappear; see Figure 1(d).

\section{Conclusion}

The generalized Buneman dispersion relation (42) was derived for two-component plasma in longitudinally dominated magnetic field. The plasma was treated as nonrelativistic under the assumption of weak perpendicular component of the magnetic field (gyration is negligible) but the resulting relation is valid for other field configurations as well. The whole calculation was done as usually in lowfrequency limit. It describes the behavior of plasmas with macroscopic particle motions (beam-target systems, two beams, etc.) in both nonzero magnetic and electric fields. The derived dispersion relation yields completely new branches of both real and imaginary frequencies, which were found numerically. It can be helpful for the stability analyses; furthermore it can be used in various tests of numerical plasma magnetohydrodynamics and hybrid codes.

\section{Acknowledgment}

This research has been supported by the Grant IAA101210801: "Simulations of the DD fusion reactions" of the Grant Agency of the Academy of Sciences of the Czech Republic.

\section{References}

[1] T. J. M. Boyd and J. J. Sanderson, The Physics of Plasmas, Cambridge University Press, Cambridge, UK, 2003.

[2] A. Bret, "Weibel, two-stream, filamentation, oblique, bell, buneman...which one grows faster?" Astrophysical Journal, vol. 699, no. 2, pp. 990-1003, 2009.

[3] I. A. Bush, A. P. L. Robinson, R. Kingham, and J. Pasley, "Cavitation and shock wave formation in dense plasmas by relativistic electron beams," Plasma Physics and Controlled Fusion, vol. 52, no. 12, Article ID 125007, 2010.

[4] O. Buneman, "Instability, turbulence, and conductivity in current-carrying plasma," Physical Review Letters, vol. 1, no. 1, pp. 8-9, 1958.

[5] O. Buneman, "Dissipation of currents in ionized media," Physical Review, vol. 115, no. 3, pp. 503-517, 1959.

[6] S. Iizuka, K. Saeki, N. Sato, and Y. Hatta, "Buneman instability in a bounded electron beam-plasma system," Journal of the Physical Society of Japan, vol. 54, no. 1, pp. 146-151, 1985.

[7] A. Hirose, "On amplitude saturation of Buneman instability and anomalous resistivity," Plasma Physics, vol. 20, no. 5, article 008, pp. 481-485, 1978.

[8] R. A. Treumann, "Origin of resistivity in reconnection," Earth, Planets and Space, vol. 53, no. 6, pp. 453-462, 2001.

[9] Y. Ohira and F. Takahara, "Oblique ion two-stream instability in the foot region of a collisionless shock," Astrophysical Journal, vol. 688, no. 1, pp. 320-326, 2008.

[10] M. Oppenheim, N. Otani, and C. Ronchi, "Saturation of the Farley-Buneman instability via nonlinear electron $E \times B$ drifts," Journal of Geophysical Research, vol. 101, no. 17, pp. 273-286, 1996.

[11] S. Iizuka, K. Saeki, N. Sato, and Y. Hatta, "Buneman instability, pierce instability, and double-layer formation in a collisionless plasma," Physical Review Letters, vol. 43, no. 19, pp. 14041407, 1979.

[12] E. S. Weibel, "Spontaneously growing transverse waves in a plasma due to an anisotropic velocity distribution," Physical Review Letters, vol. 2, no. 3, pp. 83-84, 1959.

[13] L. Landau, "On the vibration of the electronic plasma," Journal of Physics, vol. 10, no. 25, 1946.

[14] F. F. Chen, Introduction to Plasma Physics and Controlled Fusion, Springer, Berlin, Germany, 2004.

[15] T. H. Stix, Waves in Plasmas, Springer, Berlin, Germany, 2006.

[16] C. K. Birdsall and A. B. Langdon, Plasma Physics via Computer Simulation, McGraw-Hill Book, 1985.

[17] O. P. Hastings and E. Liang, "2.5 dimensional particle-in-cell simulations of relativistic plasma collisions," http://arxiv.org/ abs/0803.1201.

[18] J. Hubbard, D. Schleicher, and S. Sutherland, "How to find all roots of complex polynomials by Newton's method," Inventiones Mathematicae, vol. 146, no. 1, pp. 1-33, 2001. 

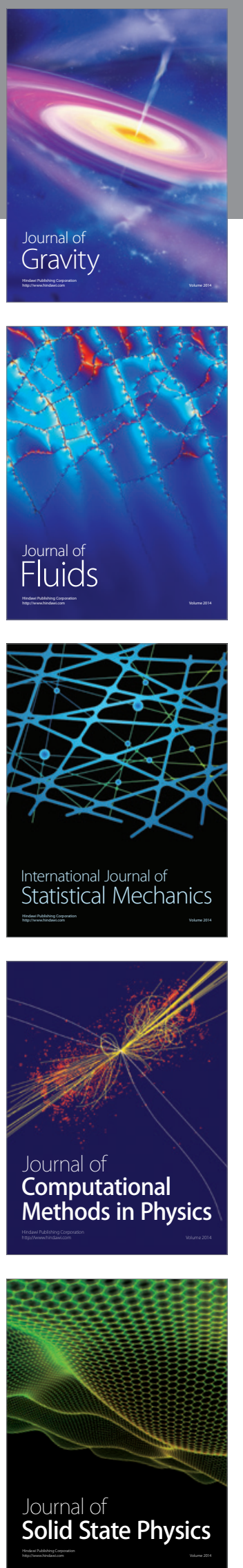
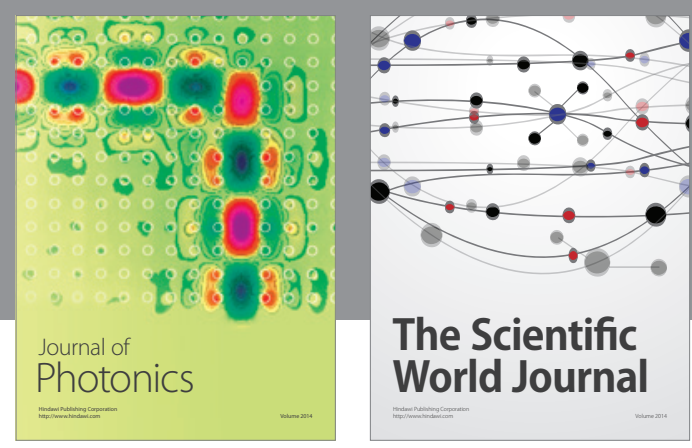

The Scientific World Journal

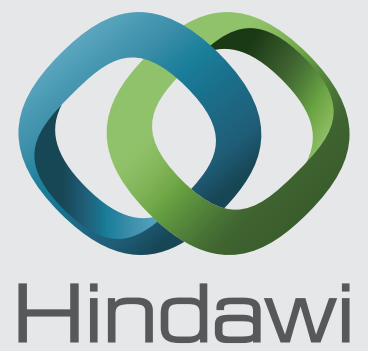

Submit your manuscripts at http://www.hindawi.com
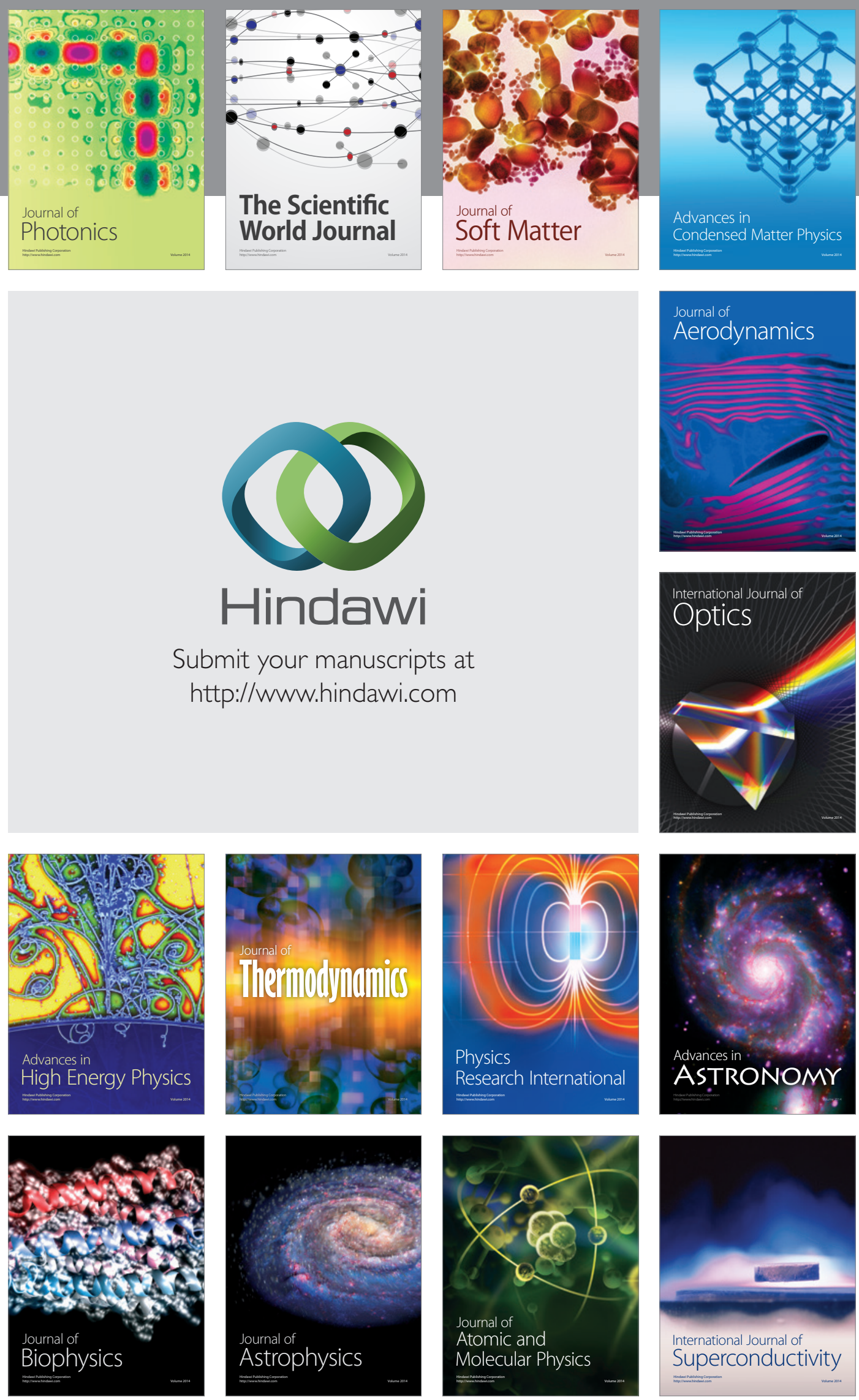
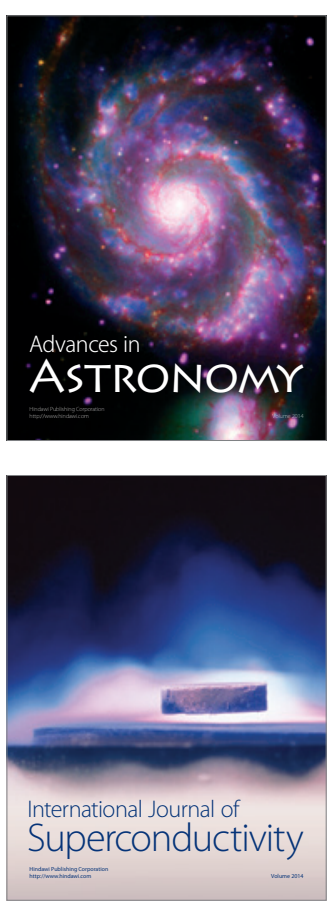